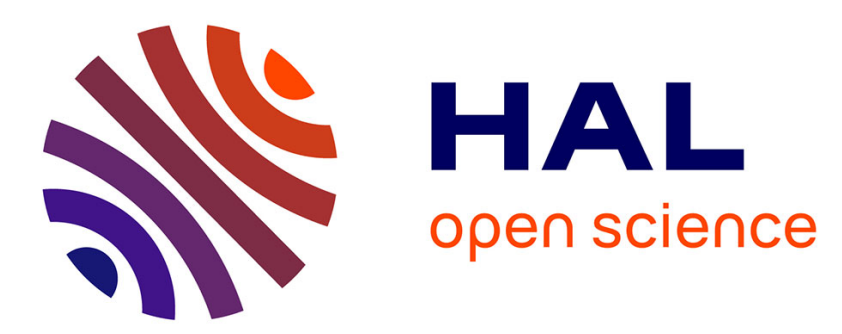

\title{
L'intonation juste dans la théorie ancienne de l'Inde: ses applications aux musiques modale et harmonique.
}

\author{
E. James Arnold, Bernard Bel
}

\section{To cite this version:}

E. James Arnold, Bernard Bel. L'intonation juste dans la théorie ancienne de l'Inde: ses applications aux musiques modale et harmonique.. Revue de musicologie, 1985, 71e (1-2), pp.11-38. hal-00373725

\section{HAL Id: hal-00373725 \\ https://hal.science/hal-00373725}

Submitted on 7 Apr 2009

HAL is a multi-disciplinary open access archive for the deposit and dissemination of scientific research documents, whether they are published or not. The documents may come from teaching and research institutions in France or abroad, or from public or private research centers.
L'archive ouverte pluridisciplinaire HAL, est destinée au dépôt et à la diffusion de documents scientifiques de niveau recherche, publiés ou non, émanant des établissements d'enseignement et de recherche français ou étrangers, des laboratoires publics ou privés. 
ARNOLD, E.J. L'intonation juste dans la théorie ancienne de l'Inde : ses applications aux musiques modale et harmonique. Revue de Musicologie, vol. 71e, no. 1-2. 1985, p. 11-38. (Edition et traduction anglais/français : Bernard BEL)

\section{L'intonation juste \\ dans la théorie ancienne \\ de l'Inde : \\ ses applications \\ aux musiques modale \\ et harmonique ${ }^{1}$}

\section{AVANT-PROPOS}

\section{Résumé}

La théorie musicale ancienne de l'Inde, exposée intégralement dans les premiers traités sanscrits sur la musique ${ }^{2}$, et qui s'intéresse à la notion de consonance, aux échelles musicales et à leurs arrangements systématiques préservant les différences enharmoniques entre les positions tonales des notes, constitue la formulation la plus incisive du système d'intonation juste qui ait jamais été proposée. Elle est à même de fournir une quantité considérable d'informations utilisables dans la pratique de la musique modale, aussi bien que dans celle de la musique harmonique.

1. Ces travaux ont bénéficié du soutien de l'American Institute of Indian Studies (Chicago) et de JDR IIIrd Fund (New York) pendant les années 1973-1975 et 1978-1979 respectivement. L'auteur tient d'autre part à remercier certaines personnes sans qui cette étude n'aurait pu être menée à bien : Lal Mani Mishra, Dr. Prem Lata Sharma, Amiyanath Bhattacharya, Pt. Hanuman P. Mishra, Pt. Dilip Chandra Vedi, Ustad R. Fahimuddin Dagar, MM. Jacques Dudon, Bernard Bel, Pierre-Yves Asselin, et ses parents qui n'ont cessé de l'aider dans les moments difficiles.

2. Nätyashāstra (IV ${ }^{\mathrm{c}}$ s. avant J.-C.), Dattilam (date incertaine) et Brihaddeshi (VII ${ }^{e} \mathrm{~s}$. après J.-C.). 
Cet article propose un modèle mathématique du système gramamurcchana (échelles fondamentales et leurs transformations modales) qui, une fois découpé et assemblé, constitue un disque à calculer circulaire d'un intérêt considérable pour l'étude des échelles musicales. Ce modèle a été conçu pour la musique modale de l'Inde, mais il peut être adapté facilement à la musique harmonique.

Cette théorie ancienne de géométrie musicale est le point de départ idéal de toute recherche sur le phénomène de l'intonation de la musique classique du Nord de l'Inde. Non seulement elle propose un arrangement enharmonique logique des échelles des ragas, en accord avec les exigences des mouvements mélodiques, mais on peut encore montrer que cette théorie fournit la clé d'une explication cohérente (fondée sur des phénomènes psycho-acoustiques simples) de la pratique traditionnelle consistant à interpréter chaque raga à une heure particulière.

De plus, le système grama-murcchana permet de répondre à certaines questions relatives à l'intonation juste dans le contexte de la musique harmonique classique occidentale, car il établit les critères de choix des positions enharmoniques des notes en fonction des enchaînements d'accords et des modulations.

\section{Perspective historique}

Bien que les traités sanscrits rédigés plus tard aient continué, pour la plupart, à décrire le système ancien, celui-ci semble avoir cessé d'être interprété clairement, déjà peut-être au XIII ${ }^{\mathrm{c}}$ siècle à l'époque du Sangītaratnäkara de Sārangadeva ${ }^{3}$. Les bouleversements culturels provoqués par les invasions musulmanes ont certainement contribué à accélérer ce processus, mais il est curieux de constater, toutefois, que la ligne de recherche qui a réussi à supplanter le système ancien est originaire du Sud de l'Inde (Swaramelakalānidhi de Rāmāmātya, 1550 après J.-C.) ${ }^{4}$.

Cette nouvelle théorie, basée sur l'idée de classer des ragas à l'aide de plusieurs (10 à 72) échelles types (mela-s) inclut, il est vrai, et pour des raisons historiques tout à fait acceptables, un grand nombre d'échelles musicales qui ne sont pas décrites par le système ancien grama-murcchana (dont la base du système de classification des entités mélodiques est constituée seulement de deux échelles fondamentales (grama-s). Mais elle renonce à prendre en considération l'idéal de l'équilibre interne des consonances mutuelles entre les deux tétracordes des échelles musicales, et par conséquent elle ne donne aucune indication sur les positions enharmoniques de leurs notes.

A partir de la découverte de l'existence du système ancien par Sir

3. O. Thakur, Pranava-bharati (Banaras: chez l'Auteur, 1956), p. 64-65.

4. P. L. Sharma, "The ancient grama system and its distortion in the medieval times ", Journal of the Music Academy, XXXII (1961), 37-40. 
William Jones ${ }^{5}$, de nombreux chercheurs ont tenté de reconstruire et d'interpréter cette théorie en l'absence d'une tradition vivante qui fasse autorité ${ }^{6}$. Pour projeter quelque lumière sur ce problème, deux siècles de recherches et de spéculations théoriques ont été nécessaires, auxquelles ont participé les plus éminents spécialistes indiens et européens. La reconstitution du système grama-murcchana est le fruit de la rencontre des deux cultures, étant donné que tout intérêt pour les questions d'échelles musicales en intonation juste était suspendu en Europe comme en Inde - mais pour des raisons différentes. Ce n'est que dans les dernières décennies, toutefois, que cette reconstitution a pu être achevée?

\section{Hypothèses de la présente étude}

L'étude qui suit montre que si l'on considère que l'ensemble de 23 positions tonales d'un cycle complet $^{8}$ de quartes et de quintes construit à partir d'une seule note de base (Do) ou $\mathrm{Sa}^{9}$ correspond au système de 22 shruti $i \mathrm{~s} \mathrm{~s}^{10}$ qui divise l'octave selon le système ancien, et si l'on établit les échelles fondamentales Sa-grama et Ma-grama, à partir de ces positions tonales en suivant les instructions des textes, les transformations de ces échelles fondamentales en différents modes (murcchana-s) (obtenues en déplaçant la tonique sur les différentes notes des grama-s) aboutissent alors à un ensemble acoustiquement idéal de positions enharmoniques des notes des échelles diatoniques, des modes chromatiques, et des accords enharmoniques de l'échelle chromatique qui leur est associée.

La qualité principale de ces échelles définies par le système gramamurcchana réside dans l'équilibre de leurs consonances mutuelles entre les deux tétracordes, équilibre mis en valeur par le jeu des mouvements mélodiques. Nous verrons que les gammes majeure et mineure de Zarlino font partie de cet ensemble. Il est intéressant de remarquer que M. Klarenz Barlow a montré récemment que l'analyse sur ordinateur de "l'harmonicité" des accords enharmoniques des

5. Sir W. Jones, On the Musical Modes of the Hindoos (1784), reproduit dans Raja S. M. Tagore, Hindu Music from Various Authors (Calcutta: Chowkhamba Sanskrit Series Office, Varanasi, 1875).

6. R. S. Bhandarkar, "Contributions to the study of ancient Hindu music", The Indian Antiquary, 1912; E. J. Arnold, «A perspective on the problem of raga shrutis and grama-murcchana analysis", Journal of the Sangeet Natak Academy (New Dehli : Sangeet Natak Academy, janvier 1981).

7. F. Framji, The Theory and Practice of Indian Music (Poona, 1938).

8. Nous remplaçons certains rapports pythagoriciens complexes par des rapports harmoniques simples. Par exemple, le rapport 8192/6561 est remplacé par 5/4, tierce majeure harmonique.

9. En ce qui concerne la musique modale, le nom des notes s'entend en valeur relative.

10. Nous examinons plus loin la raison d'être des nombres 22 et 23. 
échelles diatoniques aboutit à un choix préférentiel de positions tonales qui correspondent à celles du système grama-murcchana ainsi interprété 11 .

\section{Limites de notre étude}

Cette étude se limite au cadre de la "géométrie musicale" sans prétendre fournir des réponses définitives sur l'utilisation adéquate des intervalles musicaux. Il se peut que des positions tonales qui semblent théoriquement valables ne soient pas acceptées comme telles par l'oreille, par exemple en présence d'un générateur de tonique riche en sons harmoniques. Nous avons effectué récemment des expériences avec quelques musiciens indiens experts utilisant un tanpura et le "shruti harmonium ", orgue électronique polyphonique programmable de grande précision réalisé par M. Bernard Bel; ces expériences ont montré que l'oreille semble préférer des positions tonales diminuées de 4 ou 5 schismas $^{12}$ pour la tierce et la sixte majeures pythagoriciennes, et de 2 ou 3 pour les positions de la plupart des notes mineures, alors que les notes sensibles semblent subir plus ou moins l'attraction de leur note de résolution.

\section{UN MODĖLE MATHÉMATIQUE DU SYSTĖME SHRUTI-SWARA-GRAMA-MURCCHANA}

\section{Introduction}

Cette partie est consacrée à la description de notre modèle mathématique du système d'acoustique musicale shruti-swara-gramamurcchana ${ }^{13}$ proposé par Bharata (Nātyaśāstra, Ch. 28, 21 - 36), Dattila (Dattilam 1, 6A - 30A), Sārangadeva (Sangītaratnākara, $3: 8$ - $5: 11$ ), etc. L'idée de base consiste à monter un disque mobile gradué selon les positions tonales des échelles fondamentales ( $\mathrm{ra} a-$ $m a-s)$ sur un graphe circulaire logarithmique représentant la division de l'octave en shruti-s. Il faut noter que le terme sanscrit "shruti» est utilisé indifféremment pour désigner tantôt une position enharmonique, tantôt un intervalle ${ }^{14}$. Dans le premier cas, shruti désigne la position tonale d'une note de l'octave par rapport à la tonique

11. K. Barlow, «Bus journey to Parametron», Feedback Papers, 21-23 (Cologne: Feedback Studio, décembre 1980), 115.

12. 1 schisma $=0,84$ savart.

13. "Position enharmonique-note-échelle fondamentale-transposition modale $\gg$.

14. M. Lath, A Study of Dattilam (New Delhi: Impex India, 1978), p. 203-206. 
(note de départ, Sa ou Do). Dans le second cas, le terme shruti désigne un intervalle qui, selon le cas, peut prendre trois valeurs distinctes (voir II.2).

Pour construire ce disque à calculer, on découpera le disque mobile imprimé page 33 et on le collera sur du bristol; on collera de même sur un support rigide le graphe représenté page 35. On assemblera ces deux parties à l'aide d'une attache ou d'un rivet tubulaire permettant la rotation de la partie mobile.

Le modèle permet d'observer immédiatement les relations enharmoniques qui caractérisent la structure des échelles musicales dans le système d'intonation juste. L'erreur due au schisma est parfois visible, mais doit être ignorée. En positionnant n'importe quel swara (note) du disque mobile (disque du grama) sur le Sa (Do) du graphe des shruti-s, on obtient immédiatement la liste des positions enharmoniques des notes (shruti-s) qui composent l'échelle modale (murcchana) construite à partir de la note choisie sur le disque mobile.

En fait, les principales échelles types employées par la musique classique Hindustani forment une série principale qu'on peut déduire directement de la structure harmonique des échelles fondamentales (grama-s). Cette propriété est résumée dans la table de la "série étendue des murcchana-s " (modes) relative aux échelles qui dérivent de l'échelle fondamentale de Fa (Ma-grama) et celle de Do (Sagrama). Par "échelle fondamentale " nous entendons une structure particulière de notes, notion qu'il faut distinguer de celle "d'échelle type » (murcchana, mela, thaat) ou «mode». Le mode est simplement l'application de la structure obtenue par transposition de la tonique (Sa). Les deux échelles fondamentales citées sont deux formes desquelles découle le mode dorien; elles diffèrent seulement d'un intervalle microtonal pour la position de la note du cinquième degré du grama $\left(\mathrm{Pa}^{3}\right.$ ou $\left.\mathrm{Pa}^{4}\right)$.

\section{Description de la partie fixe du modèle (page 35)}

La couronne circulaire représente l'octave divisée selon le système des shruti-s, déterminés, au schisma près, par les cycles de quintes ascendantes et descendantes. Les symboles d'intervalles sont les suivants :

- C représente le comma syntonique $(81 / 80)$;

- M représente le demi-ton chromatique $(25 / 24)$;

- L représente le limma (256/243).

Ces intervalles indiquent la distance tonale entre les points de manifestation (sphota) des notes. Ces positions sont désignées par les abréviations des noms sanscrits des notes ( $\mathrm{Sa}, \mathrm{Ri}, \mathrm{Ga}$ etc...) suivis des exposants (1), (2), (3), (4). Les exposants (1) et (2) indiquent les deux positions enharmoniques des notes «mineures» 
(komal), et (3) et (4) celles des notes "majeures" ( tivra). Pour symboliser de manière concise la procédure à suivre pour accorder (ou définir mathématiquement) les positions de la division enharmonique de l'octave, nous avons emprunté à Jacques Dudon des symboles ainsi définis ${ }^{15}$ :

1. $\hat{\mathrm{n}}$ représente la $\mathrm{N}$-ième quinte ascendante à partir de Sa.

2. $\forall$ représente la $\mathrm{N}$-ième quinte descendante à partir de Sa.

3. $\cap$ représente la tierce majeure (harmonique) ascendante à partir de Sa.

4. $\cup \quad$ représente la tierce majeure (harmonique) descendante à partir de Sa.

5. A ou $\bigcirc$ représente la $N$-ième quinte ascendante, ou descendante à partir de la tierce majeure au-dessus de Sa.

6. $\circlearrowleft$ ou $\bigvee$ représente la $\mathrm{N}$-ième quinte ascendante, ou descendante à partir de la tierce majeure au-dessous de Sa.

3. Validité de cette division du point de vue des traités anciens

Aucun traité ancien ne fournit en fait d'indication mathématique sur la valeur d'un shruti. Ces traités se contentent de mentionner le nombre de shruti-s qui séparent deux swara-s (notes) dans les échelles fondamentales (grama-s), ainsi que les rapports de quinte entre les notes des grama-s le cas échéant. La validité traditionnelle de la division proposée s'appuie sur trois arguments :

1. La valeur acoustique des intervalles de quinte et de quarte (13 et 9 shruti-s respectivement) dans la structure harmonique des échelles est utilisée comme critère de consonance par les traités sanscrits. Les positions que nous avons établies sur le graphe appartiennent à un cycle complet de quartes et de quintes (au schisma près); ces positions divisent l'octave en vingt-deux shruti-s, comme nous verrons par la suite. Toute autre position tonale qui pourrait être proposée impliquerait l'utilisation de nouveaux axiomes plus complexes, ou introduirait encore des intervalles qui n'ont pas de signification numérique simple. Les positions utilisées dans la pratique musicale peuvent, certes, différer de ces positions théoriques, lesquelles, en fait, ne sont que des références pour l'intellect et non nécessairement pour l'oreille.

15. E. J. Arnold, «Notes on the construction of a simple shruti-swaragrama-yantra", American Institute of Indian Studies Quarterly Newsletter (New Delhi : A.I.I.S., 1974), p. 28-30. 
2. Si l'on détermine d'après notre modèle des shruti-s les échelles fondamentales (Ma et Sa grama-s) en respectant les valeurs des intervalles (en shruti-s) indiquées par les textes ${ }^{16}$, aucune transposition modale (murcchana) ne fait intervenir une position tonale qui soit extérieure au graphe d'origine. Par conséquent, cette division de l'octave en vingt-deux shruti-s, et le système des échelles fondamentales Ma-grama et Sa-grama qui en découle, forment un tout cohérent et valable aussi bien du point de vue acoustique que géométrique.

3. Il reste enfin à montrer que les vingt-trois intervalles formés par le graphe constituent en fait un système de "vingt-deux shru$t i-\mathrm{s}$ ». L'expérience des "deux vina-s » (luths) mentionnée par Bharata ${ }^{17}$ permet d'apprécier auditivement les intervalles de 4 shruti-s, 3 shru$t i$-s, et 2 shruti-s, et d'établir les équivalences respectives de ces intervalles avec le ton majeur $(9 / 8)$, le ton mineur (10/9), et le demiton diatonique (16/15) connus en Occident. De même, à partir des deux grama-s, elle permet d'apprécier auditivement les valeurs d'un comma (dans le premier abaissement d'un shruti), d'un limma (dans le deuxième abaissement d'un shruti), d'un demi-ton chromatique (dans le troisième abaissement d'un shruti), et d'un comma une fois de plus (dans le quatrième abaissement d'un shruti) ${ }^{18}$. Le «shruti» lui même n'est donc pas un intervalle de dimension fixe ${ }^{19}$.

Il faut remarquer que le limma (256/243) est égal à un demi-ton chromatique (25/24) plus un comma (81/80). C'est ici que se situe l'ambiguité entre les nombres vingt-deux et vingt-trois: en effet, entre $\mathrm{Ma}^{1}$ (Fa) et $\mathrm{Pa}^{4}$ (Sol) du graphe fixe des shruti-s, intervalle d'un ton majeur (4 shruti-s comme on le voit sur le disque mobile des grama-s), le graphe fait apparaître cinq positions enharmoniques $(\mathrm{C}+\mathrm{M}+\mathrm{C}+\mathrm{M}+\mathrm{C})$, là où il faudrait compter quatre shruti-s $(\mathrm{C}+\mathrm{M}+\mathrm{C}+\mathrm{L}$, ou bien: $\mathrm{L}+\mathrm{C}+\mathrm{M}+\mathrm{C})$.

\section{Description du disque mobile (disque de grama, page 33)}

La périphérie du disque de grama est divisée en douze notes qui constituent une gamme chromatique particulière. Cette gamme est établie d'après les principes suivants: étant données vingt-deux positions enharmoniques pour accorder les onze notes de la gamme

16. D'après les traités, il y a 3-4-2-4-3-4-2-4 shruti-s entre les notes SaRe-Ga-Ma-Pa-Dha-Ni du Ma-grama et 3-4-2-4-3-2-4 shruti-s entre les mêmes notes du Sa-grama (voir Dattilam, 1.11A-15B).

17. Bharata, Nätyasâstra, commenté par Abhinavagupta, édité par M. Ramakrishna Kavi, IV, Gaekwad's Oriental Series, $n^{\circ} 145$ (Baroda, 1964), ch. $28: 26$.

18. A. A. Bake, "Bharata's experiment with two vinas", Bulletin of the School of Oriental and African Studies, XX (London: The School of Oriental and African Studies, 1957), p. 61-67.

19. Cf. K. B. Deval, Music of East and West Compared (Poona, 1908). 
chromatique - excluant la base (Do), ou Sa, qui reste fixe - il existe $2^{\text {ll }}$, soit 2048 façons différentes, pour accorder la gamme chromatique. La plupart d'entre elles, cependant, comportent plusieurs quintes dissonantes (quintes du loup, 40/27). Douze d'entre elles sont optimales du point de vue de la consonance (ayant une seule quinte du loup). La gamme chromatique choisie sur la périphérie du disque est la seule parmi les douze qui puisse contenir toutes les positions enharmoniques de l'échelle fondamentale de ( $\mathrm{Fa}), \mathrm{Ma}-$ grama, à laquelle les traités sanscrits font référence. C'est pourquoi nous l'appellerons «la gamme chromatique Ma-grama ». Cette gamme est définie par un tour complet du cycle des quartes (en négligeant le problème du schisma).

L'échelle fondamentale de (Do), Sa-grama, ne diffère de celle de Ma-grama que par la position du cinquième degré du grama $\left(\mathrm{Pa}^{4}\right.$ au lieu de $\left.\mathrm{Pa}^{3}\right)$. Cette position $\left(\mathrm{Pa}^{4}\right)$ est indiquée sur la périphérie, de même que les positions cyuta $\mathrm{Sa}(\mathrm{Do}-\mathrm{lc}$.) et cyuta $\mathrm{Ma}(\mathrm{Fa}-\mathrm{lc}$.) qui ne font pas partie de la gamme chromatique Ma-grama mais qui sont utilisées dans la procédure dite «transposition d'un comma » (sadharana) (voir III.3).

Le deuxième cercle vers l'intérieur indique les notes des échelles fondamentales diatoniques Ma-grama et Sa-grama. Les notes non altérées (shuddha swara-s) sont marquées par des rayons en trait continu, et les notes altérées (vikrita swara-s) en pointillé. Ces notes fonctionnent principalement comme variantes des premières dans certaines phrases mélodiques où la note altérée est utilisée dans le mouvement ascendant, et la note naturelle dans le mouvement descendant ${ }^{20}$.

Nous avons indiqué sur la couronne intérieure les nombres de shruti-s qui justifient la position des notes des grama-s selon les textes ${ }^{21}$.

\section{Harmonie interne des échelles fondamentales (grama-s)}

L'harmonie interne, ou structure acoustique, des deux échelles fondamentales (grama-s) est soulignée dans le plus petit cercle du disque mobile. L'échelle fondamentale de $(\mathrm{Fa})$ et celle de (Do) (Ma et Sa grama-s) sont composées de deux chaines de consonances de quintes qui sont décalées l'une de l'autre d'une tierce majeure harmonique.

Il est à noter que l'échelle fondamentale de (Do), Sa-grama, possède cette propriété caractéristique que son cinquième degré $\left(\mathrm{Pa}^{4}\right)$ appartient à la même chaîne de consonances que (Do) $\mathrm{Sa}$, alors que dans l'échelle fondamentale de (Fa), Ma-grama, le cinquième degré $\left(\mathrm{Pa}^{3}\right)$ appartient à l'autre chaîne. Le cinquième degré, qui, de toute

20. Cf. Bharat, op. cit., $21: 35-36$.

21. Cf. note 16 . 
façon, doit figurer dans toute échelle fondamentale, doit être choisi parmi ces deux possibilités. Du point de vue théorique, ces deux choix sont également valides, et cette note constitue donc le "point critique" de l'harmonie interne des deux échelles.

Si le cinquième degré du grama appartient à la chaîne de (Do) $\mathrm{Sa}$, (cas de Sa-grama) alors les notes (Do), (Ré), (Mib), (Fa), ou $\mathrm{Sa}, \mathrm{Re}^{3}, \mathrm{Ga}^{1}, \mathrm{Ma}^{1}$, sont en consonance mutuelle avec ( $\mathrm{Sol}$ ), (La), (Sib), $\left(\mathrm{Do}_{2}\right)$ ou $\mathrm{Pa}^{4}, \mathrm{Dha}^{3}, \mathrm{Ni}^{1}, \mathrm{Sa}$. Cette échelle est formée de tétracordes «disjoints", entre (Fa) et (Sol) ou $\mathrm{Ma}^{1}$ et $\mathrm{Pa}^{4}$ et « conjoints" au (Do), Sa.

$\mathrm{Si}$, en revanche, (Sol), $\mathrm{Pa}^{3}$ appartient à la chaîne de (Ré), $\mathrm{Re}^{3}$, (cas de Ma-grama) alors les notes (Ré), (Mib), (Fa), (Sol), ou $\mathrm{Re}^{3}$, $\mathrm{Ga}^{1}, \mathrm{Ma}^{1}, \mathrm{~Pa}^{3}$, sont en consonance mutuelle avec ( $\left.\mathrm{La}\right),(\mathrm{Si} b),\left(\mathrm{Do}_{2}\right)$, $\left(\mathrm{Re}_{2}\right)$, ou $\mathrm{Dha}^{3}, \mathrm{Ni}^{1}, \mathrm{Sa}, \mathrm{Re}^{3}$. Cette échelle est formée de tétracordes disjoints entre (Sol) et $(\mathrm{La})$ ou $\mathrm{Pa}^{3}$ et $\mathrm{Dha}^{3}$, et conjoints au (Ré), $\mathrm{Re}^{3}$. Évidemment, la différence entre ces deux cas réside dans le déplacement de l'équilibre harmonique, ce qui implique différentes options de mouvement mélodique.

Nous touchons ici un phénomène extrêmement important quant aux relations entre le squelette harmonique et le mouvement mélodique des ragas. De manière générale on peut affirmer que deux phrases mélodiquement parallèles (et de morphologies rythmiques similaires) impliquent des relations de consonance obéissant au même parallélisme. Cependant, dans le cas où ces parallélismes ne sont pas formels, le système de relations entre le mouvement mélodique et la structure harmonique est plus subtil, et seule une étude expérimentale de la pratique musicale permettra d'approfondir théoriquement ces questions. Cette approche nouvelle est au cœur de nos recherches actuelles ${ }^{22}$.

Toutefois le génie du système ancien d'analyse des échelles musicales réside dans le fait que le choix crucial de la note située à la position critique (Sol) ou Pa des échelles fondamentales (grama-s) se retrouve par le jeu de transposition modale (murcchana) à des points critiques de toutes les échelles diatoniques (et leurs extensions chromatiques) reliées par la même séquence, et qui forment les souches des grandes familles de ragas.

6. L'application du système grama-murcchana à la musique hindustani contemporaine

Les principales échelles types qui sont communément reconnues dans la musique classique du Nord de l'Inde appartiennent à la logique du système grama-murcchana. Les modes (thaat ou mela) diatoniques de la musique hindustani actuelle : kalyan, bilaval, khamaj,

22. E. J. Arnold et G. B. Bel, Étude acoustique des structures modales et mouvements mélodiques (Bourges : chez les Auteurs, 1980), p. 9 et 21. 
DE LA MUSIQUE HINDUSTANI CONTEMPORAINE

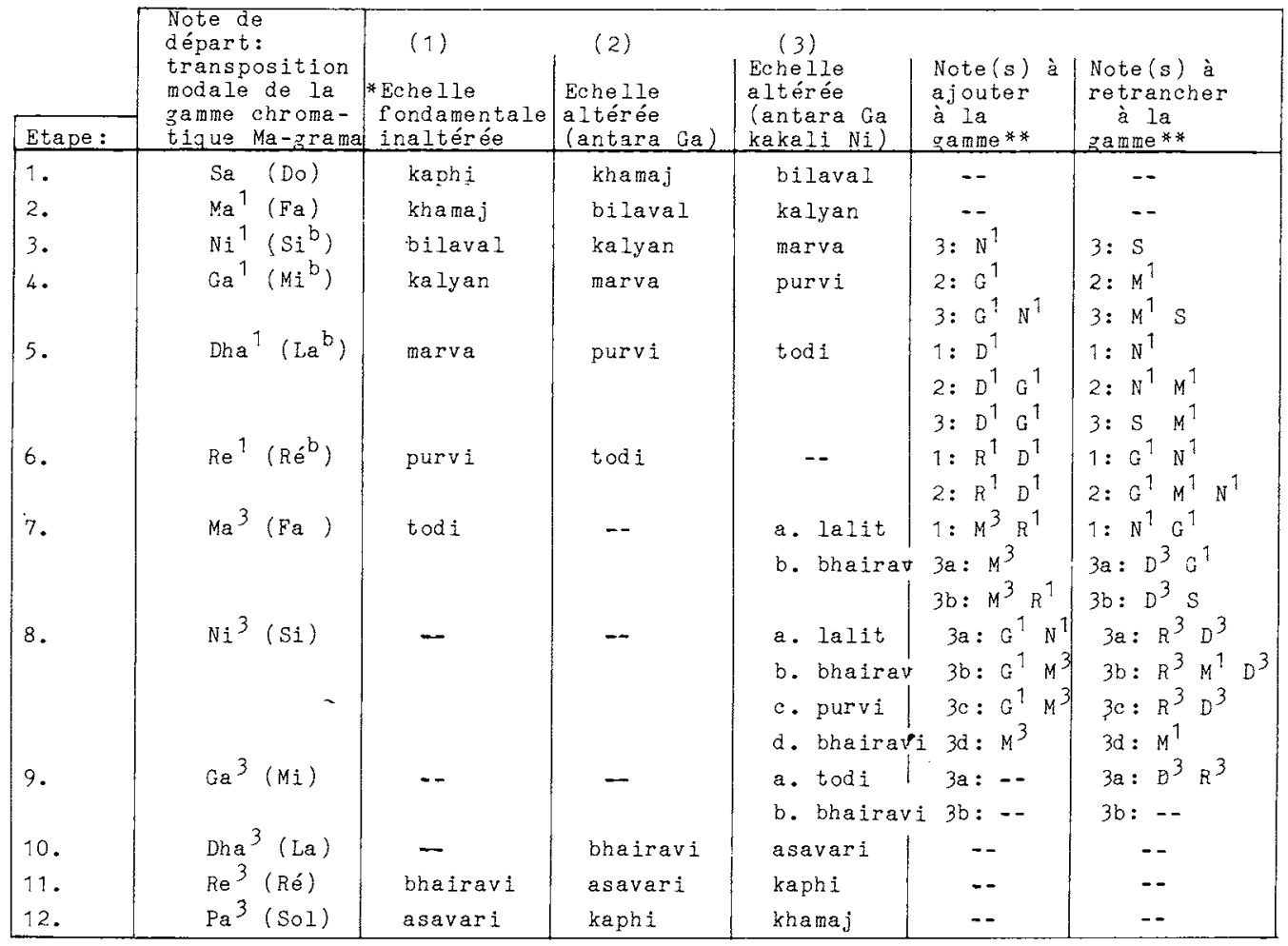

* Le terme "échelle fondamentale " désigne indifféremment l'échelle Sa-grama ou l'échelle Ma-grama.

** Les nombres renvoient aux colonnes (1), (2) et (3).

kaphi, asavari, et bhairavi (équivalant respectivement aux modes de (Fa), (Do), (Sol), (Ré), (La) et (Mi) sur les touches blanches du piano) sont les échelles types (murcchana-s) qui commencent respectivement par les notes des échelles fondamentales: $\mathrm{Ga}^{1}, \mathrm{Ni}^{1}$, $\mathrm{Ma}^{1}, \mathrm{Sa}, \mathrm{Pa}^{3}$ et $\mathrm{Re}^{3}$. (Nous conseillons au lecteur d'utiliser le modèle circulaire pour se rendre compte de ce mécanisme.) Les échelles types qui contiennent un ou deux intervalles de seconde augmentée, marva, purvi, todi, lalit, et même bhairao dans une certaine mesure, appartiennent à une extension du premier ordre des paramètres du système grama-murcchana ${ }^{23}$.

Pour relier ces échelles types à la logique du grama, il est nécessaire de relâcher quelque peu les règles de l'ancien système. En

23. Ces échelles sont appelées "modes chromatiques" par la tradition grecque. 
premier lieu, il faut utiliser les murcchana-s commençant par les notes altérées (vikrit swara-s: $\mathrm{Ga}^{3}$ et $\mathrm{Ni}^{3}$ ). De plus, il faut accepter des murccha-na-s débutant sur une note extérieure aux grama-s, mais appartenant à l'échelle que nous avons acceptée comme une extension logique de l'échelle de base: l'échelle chromatique Ma-grama (voir III.3). Il est nécessaire, enfin, de supprimer quelques notes non altérées des grama-s lorsqu'elles sont remplacées par les notes altérées ${ }^{24}$.

Le tableau ci-contre, qui résume la séquence principale des échelles types du système grama-murcchana étendu à ce qui précède, montre que les modes principaux (thaat-s) de la musique du Nord de l'Inde constituent une suite logique d'échelles types dont l'harmonie interne découle de celle des grama-s.

Pour les. murcchana-s débutant sur chaque note de l'échelle chromatique Ma-grama, le tableau indique quel mode (thaat) est produit :

1. à partir de l'échelle fondamentale non altérée;

2. à partir de l'échelle fondamentale altérée avec antara gandhara $\left(\mathrm{Ga}^{3}\right)$;

3. à partir de l'échelle fondamentale altérée avec antara grandhara $\left(\mathrm{Ga}^{3}\right)$ et kakali nishad $\left(\mathrm{Ni}^{3}\right)$.

Le tableau indique enfin les notes qui sont ajoutées et celles qui, le cas échéant, sont supprimées de l'échelle fondamentale.

\section{L'HEURE D'INTERPRÉTATION DES RAGAS ET LE SYSTÈME GRAMA-MURCCHANA}

\section{Introduction}

L'idée qu'on puisse associer divers types mélodiques avec les heures de la journée qui conviennent à leur interprétation, idée reposant sur la sensation que certains modes musicaux évoquent certains sentiments ou une ambiance spécifique compatible avec certaines heures de la journée ou de la nuit, est certainement l'un des faits les plus frappants de la musique classique du Nord de $1^{\prime} I n d e{ }^{25}$. Il est curieux qu'une propriété aussi insolite de ce système musical n'ait jamais été étudiée de manière approfondie ${ }^{26}$. Bien que cette association soit très ancienne - mentionnée pour la première fois

24. Il est à noter que la règle de avahashi madhyama (l'usage invariable de la note Ma des grama-s) est transgressée occasionnellement (voir Dattilam, 20A-B).

25. Voir le quatrième paragraphe de cette partie.

26. B. C. Deva, "Ragas and their time", Psychoacoustics of Music and Speech (Madras: The Music Academy, 1967), 140. 
par Matanga au VIII $^{c}$ siècle - l'essentiel de ce qui tient lieu aujourd'hui de théorie de l'association des ragas avec leurs heures d'interprétation consiste en quelques règles fragmentaires éparses et à peine ébauchées ${ }^{27}$.

Comme Deva l'a souligné, la plupart de ces règles impliquent des considérations d'échelle, de position du vadi swara (la note de tension du raga) ou encore de mouvement mélodique du raga ${ }^{28}$. Si l'on se limite aux questions des échelles types, le problème peut être approché à deux niveaux: la perspective chromatique et la perspective transiliente $^{29}$.

\section{La perspective chromatique}

En partant de la gamme chromatique Ma-grama, on retrouve par transposition modale les douze gammes chromatiques de consonance optimale. Il est logique de considérer comme début de cette série la

Majorisation PROGRESSIVE des Shruti-S

DE LA GAMME CHROMATIQUE

\begin{tabular}{|c|c|c|c|c|c|c|c|c|c|c|c|c|c|c|}
\hline \multirow{2}{*}{$\frac{\text { étape }}{1 .}$} & \multicolumn{2}{|c|}{$\begin{array}{l}\frac{\text { murcchana }}{\text { de la }} \\
\text { Ma-grama } \\
\text { chromatique } \\
\text { a partir de }\end{array}$} & \multicolumn{10}{|c|}{ 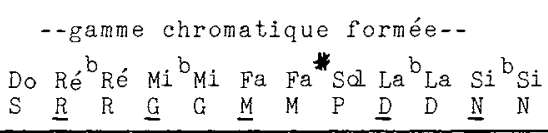 } & \multicolumn{2}{|c|}{$\begin{array}{l}\text { valeur } \\
\text { esthétique }\end{array}$} \\
\hline & $\mathrm{Sa}$ & (Do) & $\circ$ & - & - & - & - & - & - & - & - & - & plus & sombre \\
\hline 2. & $\mathrm{Ma}^{1}$ & $(F a)$ & o & - & - & - & - & - & 7 & - & - & - & & • \\
\hline 3. & $\mathrm{Ni}{ }^{1}$ & $\left(S_{i}^{b}\right)$ & $\circ$ & - & & - & - & - & + & - & - & - & & . \\
\hline 4. & $\mathrm{Ga}^{1}$ & $\left(M i^{b}\right)$ & o & - & +1 & - & - & - & + & - & 7 & - & & . \\
\hline 5. & $\operatorname{Dha}^{\uparrow}$ & $\left(L a^{b}\right)$ & $\circ$ & - & + & +1 & - & - & + & - & +1 & - & & • \\
\hline 6. & $\operatorname{Re} e^{1}$ & $\left(R e^{b}\right)$ & 0 & -7 & + & + & - & - & + & - & + & $\sqrt{t}$ & régio & $n$ de \\
\hline 7. & $\mathrm{Ma}^{3}$ & $\left(F a^{*}\right)$ & $\circ$ & - & + & +1 & - & $t$ & + & - & + & + & & a) \\
\hline 8. & $\mathrm{Ni}^{3}$ & (Si) & 0 & + & + & - & $=$ & $F$ & t & - & $-F$ & + & & . \\
\hline 9. & $\mathrm{Ga}^{3}$ & $(M i)$ & $\circ$ & $t$ & + & $\neq$ & - & t & + & + & + & + & & • \\
\hline 10. & $\mathrm{Dha}^{3}$ & (La) & $\circ$ & + & + & + & - & + & + & + & + & - & & . \\
\hline 11. & $\mathrm{Re}^{3}$ & (Ré) & $\circ$ & t & + & + & - & $t$ & + & + & + & + & & . \\
\hline 12. & $\mathrm{~Pa}^{3}$ & $(S \circ 1)$ & $\circ$ & + & + & + & $t$ & t & + & + & + & + & plus & claire \\
\hline
\end{tabular}

Légende :

$-=$ position potentielle la plus basse.

$+=$ position potentielle la plus haute.

$=$ position fixe.

27. K. N. Das, "Time theory of the ragas (North Indian)", Journal of the Music Academy, XVII (Madras: The Music Academy, 1951), 69-82.

28. Deva, art. cit., 140.

29. Nous utiliserons ce terme pour désigner une échelle musicale dans laquelle certaines notes ont été omises. 
gamme dont les douze notes occupent les positions microtonales potentielles (shruti-s) les plus basses; dans ce cas la gamme qui se retrouve en fin de série est celle dont les douze notes occupent les positions les plus hautes. La note Do (Sa) occupe évidemment toujours la même position. La première gamme est composée du cycle des quartes (Sa murcchana de l'échelle chromatique Ma-grama); la dernière est composée $\mathrm{du}$ cycle des quintes $\left(\mathrm{Pa}^{3}\right.$ murchana de l'échelle chromatique Ma-grama). Cette série de transitions ordonnées (de «mineur» vers « majeur») des shruti-s de la gamme chromatique est indiquée dans le tableau ci-contre, mise en parallèle avec sa signification esthétique.

Il est à noter que, jusqu'à la septième étape incluse, la transition n'affecte que les notes "majeures" (tivra), et qu'au-delà elle affecte seulement les notes "mineures" (komal).

La gamme chromatique de la septième étape est la plus active de cette série: elle a toutes ses notes mineures (komal) en position extrême mineure (atikomal), et simultanément toutes ses notes majeures (tivra) en position extrême majeure (tivratar). Tous les shruti-s de cette échelle particulière appartiennent à la famille pythagoricienne. Les positions microtonales pythagoriciennes sont les extrêmes limites des manifestations claires de chaque degré de l'octave et leurs rapports de fréquences sont en général plus complexes que ceux des positions harmoniques ${ }^{30}$. Ces rapports complexes impliquent plus de "tensions internes", et donc, sur le plan esthétique, une plus grande sévérité.

Il est curieux de constater que les notes mineures et majeures sont en état de tension extrême au sein de la même gamme chromatique. Les notes «froides» (les mineures) sont au plus froid alors que les notes "chaudes" (les majeures) sont au plus chaud. Cette gamme chromatique est donc une gamme de transition qui possède un degré important d'instabilité, de "stress". Ce type de transition, impliqué sur le plan esthétique par les tensions au sein des gammes chromatiques, peut être mis en parallèle avec la période de transition de l'activité diurne et nocturne: le lever et le coucher $\mathrm{du}$ soleil.

«De même que le sandhiprakasha du soir (crépuscule) représente le moment le plus chaud de la nuit, à partir duquel la nuit se rafraîchit, ainsi le sandhiprakasha du matin (aube) représente le moment le plus froid du jour, à partir duquel il se réchauffe. Par analogie, les sixième et septième étapes contiennent toutes deux les notes majeures les plus "chaudes", qui se "refroidissent" peu à peu si l'on remonte vers la première étape, aussi bien que les notes mineures les plus "froides", qui se "réchauffent" progressivement si l'on va en direction de la douzième étape."

"Ainsi nous devons nous attendre à ce que les ragas de la nuit utilisent des échelles qui, à mesure que la nuit s'avance, sont nées

30. Arnold, "Notes... », art. cit., 30 . 
des échelles chromatiques plagales qui évoluent vers la première étape. De même nous devons nous attendre à ce que les ragas du jour utilisent des échelles qui, à mesure que le jour progresse, naissent des échelles chromatiques plagales évoluant vers la douzième étape $"{ }^{31}$. Cette relation est exprimée graphiquement par le diagramme page 25 .

\section{Le point de vue des échelles transilientes}

Pour envisager la question de l'heure du point de vue des échelles transilientes de sept à neuf notes, il est nécessaire de se référer au tableau des échelles de la série étendue grama-murcchana (voir page 20). Ce tableau indique l'affinité naturelle entre les notes de la gamme chromatique Ma-grama et certaines échelles types particulières dont la structure est liée à celle des échelles fondamentales (Magrama et Sa-grama).

Nous devons donner ici quelques détails techniques que le lecteur peut négliger s'il préfère se référer tout de suite au diagramme qui résume la relation entre les heures et les échelles types reliées aux grama-s (voir page 27).

La série étendue grama-murcchana d'échelles donnée page 20 est récurrente : à partir de la douzième étape $\left(\mathrm{Pa}^{3}\right.$ murcchana), on peut en imaginer une treizième qui serait le début d'un nouveau cycle. Toutefois, du fait que nous commençons un nouveau cycle de quintes descendantes, la treizième murcchana serait construite à partir du Sa du grama abaissé d'un comma, ce qui est appellé cyuta Sa. Le lecteur pourra se reporter au modèle circulaire pour constater que cette échelle n'est autre que celle de l'étape 1 transposée vers le haut d'un comma. Nous constatons que la série "progresse", non comme un cycle fermé, mais selon une spirale. Mais pour approcher le problème de l'heure d'interprétation en relation avec la séquence principale des échelles types (la série étendue gramamurcchana), il est nécessaire d'avoir la liberté de commencer la séquence à l'étape 7 et de passer directement de l'étape 12 à l'étape 1, et vice-versa comme nous le verrons par la suite. Pour boucler le cycle, il suffit donc de procéder à ce que les textes appellent sadja-sadharana ${ }^{32}$, opération qui consiste à hausser d'un comma le cyuta Sa de l'échelle fondamentale, ce qui revient à utiliser le Sa d'origine.

En adoptant le même principe que dans le point de vue chromatique, à savoir que la septième étape (qui correspond aussi à l'échelle type la plus active) doit représenter le sandhiprakasha coucher et lever du soleil ${ }^{33}$, pendant la nuit il faut passer

31. Ibid., p. 26-27.

32. R. K. Shringy, P. L. Sharma, Sangita Ratnakara of Sarngadeva: text and translation (Bénarès: Motilal Banarsidass, 1978), vol. 1, 5: 7-9.

33. En fait le processus commence une heure avant l'aube ou le crépuscule. 


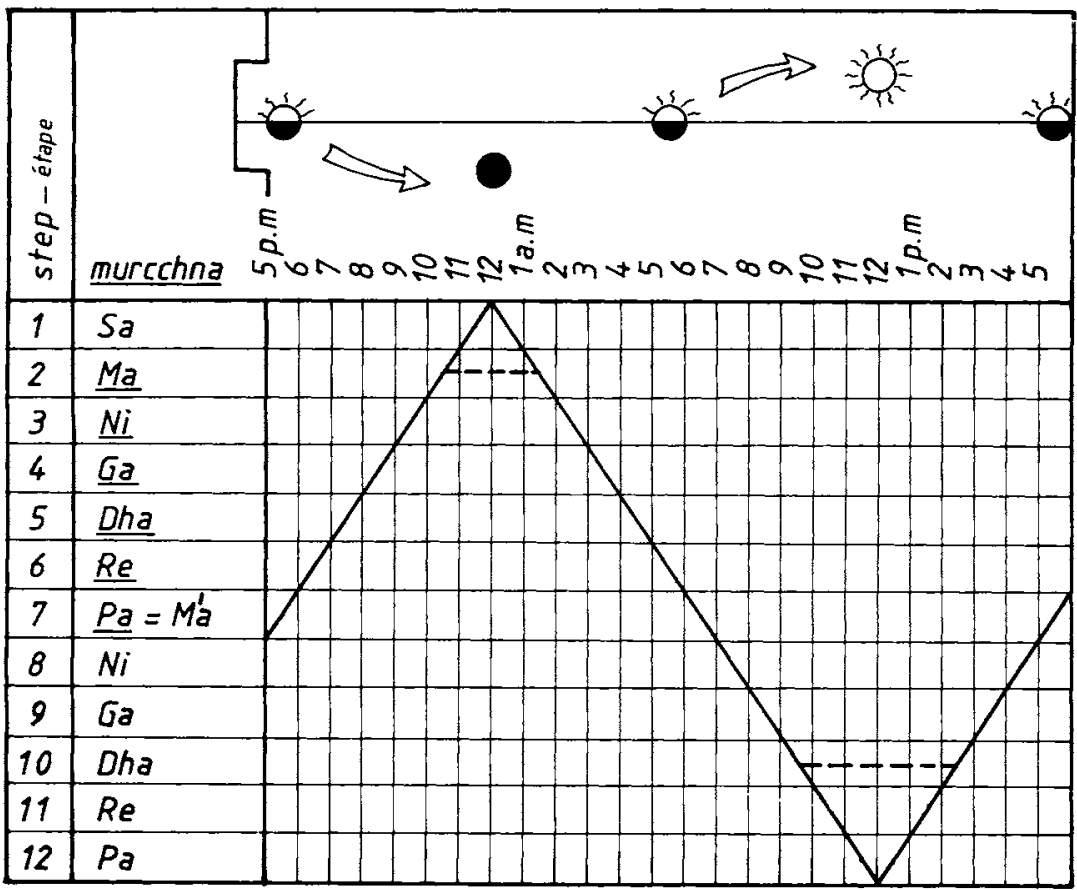

A noter que les échelles correspondant aux étapes 1 et 12 utilisent respectivement $\mathrm{Pa} 3$ et $\mathrm{Ma} 2$, qui forment des quintes et quartes imparfaites avec Sa; c'est pourquoi elles ne peuvent pas être adoptées pratiquement en présence d'un générateur de tonique accordé sur Sa (Do). D'où le tracé en pointillé.

progressivement de l'étape 7 à l'étape 1, et pendant le jour de l'étape 7 à l'étape 12. Toutefois, pour suivre l'évolution naturelle à travers les différentes échelles types, après minuit lorsque la nuit s'avance, et après midi lorsque le jour progresse, il faut pouvoir passer de l'étape 12 à l'étape 1 (et vice-versa) directement. Du point de vue chromatique cette possibilité est à rejeter du fait de la discontinuité qu'elle introduirait dans la majorisation (ou minorisation) progressive des shruti-s (voir tableau, p. 22). Mais du point de vue des échelles transilientes, la continuité entre les modes: khamaj, kaphi, asavari, bhairavi qui ont 2, 3, 4 et 5 notes mineures (komal) respectivement, est évidente. Le passage de kaphi à asavari, parfaitement logique en ce qui concerne l'évolution des échelles types et de "l'ambiance cosmique » suivant l'heure du jour (ou de la nuit), ne doit cependant pas impliquer une discontinuité (passer des murchana-s de l'échelle fondamentale à partir de $\mathrm{Sa}$, puis $\mathrm{Pa}^{3}$ ) en ce qui concerne les possibilités microtonales, comme nous l'avons déjà remarqué. 
Aussi convient-il de distinguer deux «impulsions» motrices : l'une est la continuité de l'évolution des échelles chromatiques qui soustendent les échelles types diatoniques, l'autre est celle de la séquence des échelles types reliées à l'échelle (diatonique) fondamentale (grama). Il est indispensable que ces deux évolutions progressives soient respectées lors des passages mentionnés : après minuit et après midi. Pour cela, il suffit d'accepter certaines échelles types appartenant à la série grama-murcchana, mais pour lesquelles la note de départ de leur murcchana "définitif " n'est pas la même selon que l'on considère l'échelle chromatique de base pour déterminer ses positions enharmoniques. Ainsi, par exemple, aux environs de 23 heures on devrait interpréter les ragas du mode kaphi (mode de Ré), et après minuit ceux du mode asavari (mode de La). Mais les premiers, qui sont déduits de la transposition modale à partir de Sa, ont toutes leurs notes sur les positions potentielles les plus basses, alors que les derniers, formés à partir de $\mathrm{Pa}^{3}$ murcchana, auraient toutes leurs notes sur les positions les plus hautes. Un tel accord pour asavari pourrait convenir le jour, mais certainement pas en pleine nuit!

La solution consiste donc à suivre les séries de transpositions modales de l'échelle diatonique fondamentale Ma-grama ou Sa-grama pour déterminer les échelles types, et celles de l'échelle chromatique Ma-grama en ce qui concerne les positions microtonales des notes (swara-s) de ces échelles types. C'est ce qui est résumé par le diagramme page 27 .

\section{Heures d'interprétation, murcchana-s, et repas}

Conformément à ces indications, le tableau pages 28-29, résume, pour 85 ragas environ, les heures d'interprétation, les transpositions modales (murcchana-s) correspondantes, ainsi que l'accord de leurs échelles types suivant le système des 22 shruti-s. Les notes munies de deux exposants, dont un entre parenthèses, sont celles qui résultent du choix Sa-grama/Ma-grama, et qui constituent donc le "point critique » de l'échelle (voir page 19).

Il faut considérer ces indications, non comme un système définitif, mais plutôt comme une sorte de guide: le lecteur est invité à expérimenter lui-même auditivement les implications du système grama-murcchana. Les indications d'heure d'interprétation ne doivent pas prêter à une rigidité abusive: pour les musiciens, cette question est plutôt une affaire d'intuition comme de tradition, et ses règles sont plus lâches que le tableau pourrait le laisser croire.

Pourtant, ce qui est tout à fait remarquable, c'est que les indications d'heures d'interprétation que nous avons déduites systématiquement de la théorie ancienne grama-murcchana sont presque intégralement conformes à celles que la tradition a véhiculées jusqu'à nos jours. Ceci est la preuve indubitable de l'extrême consistance et de la pertinence du système grama-murcchana, comme de la continuité du système du raga au cours des âges ! 


\begin{tabular}{|c|c|c|c|c|}
\hline 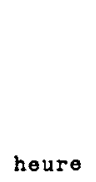 & 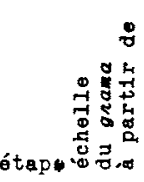 & échelles-type correspondantes & 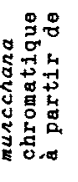 & ragas \\
\hline $6-7$ & 8. $\mathrm{N1}^{3}$ & $\begin{array}{c}\text { S R2 G1 G4 M1 M/ F4 D1(2) N1 N/4 } \\
\text { (todi/lalit/bhatrao) }\end{array}$ & $\mathrm{Ni}^{3}$ & $\begin{array}{l}\text { Vasant-mukhari } \\
\text { Ramkali } \\
\text { Ah1r-bhairao }\end{array}$ \\
\hline (ou) & 6. $\mathrm{Re}^{1}$ & 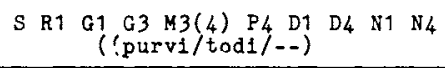 & $\operatorname{Re}^{1}$ & $\begin{array}{l}\text { Vibhas (purvi) } \\
\text { Todi }\end{array}$ \\
\hline \multirow{2}{*}{$7(\overline{o u})^{8}$} & 9. $\mathrm{Ga}^{3}$ & $\begin{array}{c}\text { S R2 G1(2) M1 M4 P4 D2 N1 N/4 } \\
(--/-- \text { /todi/bha1ravi) }\end{array}$ & $\mathrm{Ga}^{3}$ & $\begin{array}{l}\text { Gunkali } \\
\text { Vilaskhan } 1 \text { todi }\end{array}$ \\
\hline & 5. Dhe ${ }^{1}$ & $\begin{array}{c}\text { S R1 G1 G4 M3(4) P4 D1 D4 N4 } \\
\text { (marva/purvi/todi) }\end{array}$ & $\operatorname{Re}^{\prime}$ & $\begin{array}{l}\text { Ananda-bhairao } \\
\text { Bhatt1yar } \\
\text { Vibhas (marva) }\end{array}$ \\
\hline \multirow{2}{*}{$\begin{array}{r}8-9 \\
\text { (ou) }\end{array}$} & 10. $\mathrm{Dha}^{3}$ & $\begin{array}{c}\text { S R2 R4 G2 M1 M4 P4 D2 N1(2) } \\
\text { (- - /bhalravi/asavar1) }\end{array}$ & Dha ${ }^{3}$ & $\begin{array}{l}\text { Shuddha-bhairavi } \\
\text { Bhupali-todi }\end{array}$ \\
\hline & 4. $\mathrm{Ga}^{1}$ & $\begin{array}{c}\mathrm{SRT}_{4} \mathrm{R}_{4} \mathrm{G}_{4} \mathrm{M} 3(4) \mathrm{P}_{4} \mathrm{D}_{1} \mathrm{D}_{4} \mathrm{~N}_{4} \\
\text { (kalyan/marva/purvi) }\end{array}$ & $\operatorname{Re}^{1}$ & $\begin{array}{l}\text { Hindol } \\
\text { Deshkar }\end{array}$ \\
\hline \multirow{2}{*}{$9-10$} & 11. $R e^{3}$ & $\begin{array}{l}\text { S R2 R4 G2 M1(2) P4 D2 D4 N2 } \\
\text { (bhairavi/asavar1/kaph1) }\end{array}$ & $\mathrm{Re}^{3}$ & $\begin{array}{l}\text { Komal-asavar } 1 \\
\text { Bhatravi } \\
\text { Gandhara }\end{array}$ \\
\hline & 3. & $\begin{array}{c}\text { SR1 R4 G4 M1 M3(4) P4 D4 N4 } \\
\text { (bilaval/kalyan/marva) }\end{array}$ & $R$ & $\begin{array}{l}\text { Gandhara } \\
\text { Bilaval }\end{array}$ \\
\hline \multirow{2}{*}{$\begin{array}{r}10-11 \\
(\text { ou })\end{array}$} & 12. $\mathrm{Pa}^{3}$ & $\begin{array}{c}\text { S R4 G2 G4 M1 P4 D2 D4 N1(2) } \\
\text { (asavari/kaphi/khamaj) }\end{array}$ & $\mathrm{Dha}^{3}$ & \multirow{2}{*}{$\begin{array}{l}\text { 'savari } \\
\text { Devgandhar } \\
\text { Deshi } \\
\text { Bilaval }\end{array}$} \\
\hline & 2. $\mathrm{Ma}$ & 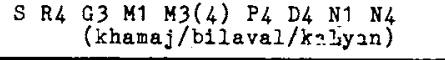 & $\mathrm{Re}$ & \\
\hline $11-12$ & 1. $\mathbf{S a}$ & $\begin{array}{c}\text { S R4 G2 G4 M1 P4 D4 N1(2) N4 } \\
\text { (kaphi/khamaj/bilaval) }\end{array}$ & Dha ${ }^{3}$ & $\begin{array}{l}\text { Deshi } \\
\text { Suha } \\
\text { Sugharal } \\
\text { Bilaval } \\
\text { Sarang }\end{array}$ \\
\hline $\begin{array}{l}\text { apres } \\
\text { midi } \\
12-1\end{array}$ & 2. $\mathrm{Ma}^{1}$ & $\begin{array}{c}\mathrm{SR}_{4} \mathrm{G}_{4} \mathrm{M1}, \mathrm{M}_{4} \mathrm{P}_{4} \mathrm{D}_{4} \mathrm{~N} 1 \text { (2) } \mathrm{N}_{4} \\
\text { (khamaj/bilaval/kalyan) }\end{array}$ & $\mathrm{Dha}^{3}$ & $\begin{array}{l}\text { Madhyamad-sarang } \\
\text { Brindavani- } \\
\text { sarang } \\
\text { Shuddha-sarang }\end{array}$ \\
\hline $1-2$ & 3. $\mathrm{Ni}^{1}$ & 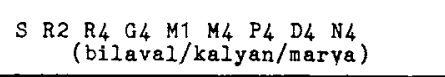 & $\operatorname{Dh} a^{3}$ & $\begin{array}{l}\text { Suddha-sarang } \\
\text { Gaud-sarang }\end{array}$ \\
\hline \multirow{2}{*}{$\begin{array}{r}2-3 \\
\text { (ou) }\end{array}$} & 2. $\mathrm{Ma}^{1}$ & 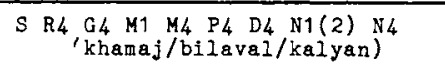 & & \multirow{2}{*}{$\begin{array}{l}\text { Suddha-sarang } \\
\text { Gaud-sarang } \\
\text { Brindivani-saran } \\
\text { Din-ki-puriya }\end{array}$} \\
\hline & 4. $\mathrm{Ga}^{1}$ & $\begin{array}{c}\text { S R2 } \begin{array}{c}\mathrm{R} 4 \mathrm{G} 4 \mathrm{M} 4 \mathrm{P} 4 \mathrm{D} 2 \mathrm{D} 4 \\
\text { (kalyan/marva/purvi) }\end{array} \\
\end{array}$ & $D h a^{3}$ & \\
\hline \multirow{2}{*}{$\begin{array}{r}3-4 \\
\text { (ou) }\end{array}$} & 1. Sa & 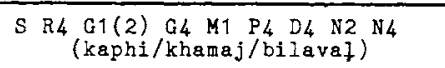 & & \multirow{2}{*}{$\begin{array}{l}\text { Bhimpalasi } \\
\text { Dhanashri } \\
\text { Din-ki-puriya } \\
\text { Madhuvanti } \\
\text { Patmanjari } \\
\text { Multani }\end{array}$} \\
\hline & 5. $\operatorname{Dh} \mathrm{s}^{1}$ & 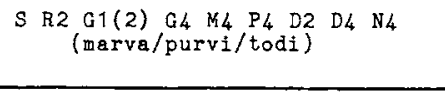 & $\mathrm{Ga}^{3}$ & \\
\hline \multirow{2}{*}{$\begin{array}{r}4-5 \\
\text { (ou) }\end{array}$} & 1. $\mathrm{Sa}$ & 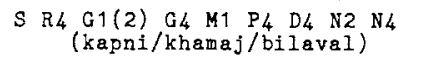 & $\mathrm{Ga}^{3}$ & \multirow{2}{*}{$\begin{array}{l}\text { Bhimpalasi } \\
\text { Patadip } \\
\text { Madhuvanti } \\
\text { Multani }\end{array}$} \\
\hline & 6. $R \mathrm{e}^{1}$ & $\begin{array}{l}\text { S R2 G1 G4 M4 P4 D1(2) N1 N4 } \\
\text { (purvi/todi/--) }\end{array}$ & $\mathrm{Ni}^{3}$ & \\
\hline
\end{tabular}

* Les chiffres entre parenthèses indiquent les positions enharmoniques qui dérivent du cinquième degré du Sa-grama (voir III.4). 


\section{APPLICATION DU SYSTÈME GRAMA-MURCCHANA A LA MUSIQUE HARMONIQUE CLASSIQUE OCCIDENTALE}

Cette étude, menée en collaboration avec M. Pierre-Yves Asselin, pendant l'été 1980, montre que le système ancien de l'Inde peut fournir la clé du problème des positions enharmoniques des notes dans les enchaînements d'accords ou modulations de tonalité.

\section{Le décalage des toniques des modes majeur et mineur}

Nous avons observé expérimentalement, à l'aide du shruti harmonium, que les gammes majeure et mineure:

$$
\begin{aligned}
& \text { Do } \left.\frac{8}{9} \text { Ré } \frac{10}{9} \mathrm{Mi} \frac{16}{15} \text { Fa } \frac{256}{243} \text { (Fa }\right) \frac{16}{15} \text { Sol } \frac{10}{9} \mathrm{La}_{8}^{9} \mathrm{Si}_{15}^{16} \mathrm{Do}_{2} \\
& \text { mineure } \\
& \text { Do } \frac{16}{15} \text { (Réb) } \frac{256}{243} \text { Ré } \frac{16}{15} \mathrm{Mib} \frac{10}{9} \mathrm{Fa} \frac{9}{8} \mathrm{Sol} \frac{16}{15} \mathrm{Lab} \frac{9}{8} \mathrm{Sib} \frac{10}{9} \mathrm{Do}_{2}
\end{aligned}
$$

qui définissent les positions relatives des notes, des accords en intonation juste, ne peuvent pas être construites sur la même gamme chromatique, et que le système d'intonation juste pour la musique harmonique (de même que pour la musique modale) utilise en fait les douze gammes chromatiques «optimalement consonantes" (qui ne contiennent qu'une quinte imparfaite) (voir II.4).

Or, la juxtaposition des gammes majeure et mineure ci-dessus n'appartient pas à une gamme chromatique de cette série. La gamme de Do (etc.) majeur, par exemple, n'est pas construite sur la même tonique que celle de Do (etc.) mineur: les premier, deuxième, et cinquième degrés (notes communes à ces deux gammes) doivent être, dans le cas de la gamme mineure, abaissés d'un comma. Par rapport $\mathrm{au}$ système dintonation juste, la gamme de Do mineur doit être déduite par transposition modale (changement de murcchana) de la gamme de Mib majeur, de même que la gamme de La mineur est une transposition modale de la gamme de Do majeur. Ainsi, pour passer de Do majeur à Do mineur, il faut moduler par quatre quintes descendantes successives (Do-Fa-Sib-Mib), puis prendre la gamme mineure relative.

L'effet de ce processus sur les positions enharmoniques des notes 
peut être montré à l'aide du modèle circulaire. On procédera ainsi :

1. Première étape : placer le $\mathrm{Ma}^{\prime} \mathrm{du}$ disque mobile sur le $\mathrm{Sa}$ (Do) de la partie fixe. Examiner quelles positions tonales de la partie fixe sont en face des repères de la partie mobile:

$$
\mathrm{Sa}-\mathrm{Re}^{3}-\mathrm{Ga}^{3}-\mathrm{Ma}^{1}-\mathrm{Pa}^{4}-\mathrm{Dha}^{3}-\mathrm{Ni}^{1}-\mathrm{Sa}
$$

(qui est le Sa-grama utilisant la note de remplacement $\mathrm{Ga}^{3}$ (antara gandhara ${ }^{34}$. Ces positions tonales sont celles de la gamme de Zarlino (Do majeur) :

$$
\mathrm{Sa}-\mathrm{Re}^{4}-\mathrm{Ga}^{3}-\mathrm{Ma}^{1}-\mathrm{Pa}^{4}-\mathrm{Dha}^{3}-\mathrm{Ni}^{3}-\mathrm{Sa}
$$

avec leurs noms indiens.

2. Deuxième étape: tourner le disque mobile d'une quinte descendante; le $\mathrm{Ma}^{1} \mathrm{du}$ disque mobile vient en face du $\mathrm{Ma}^{1}(\mathrm{Fa})$ de la partie fixe. Ainsi, la gamme de Zarlino, qui part de $\mathrm{Ma}^{\mathrm{l}}$ sur le disque mobile, a été transposée à Fa d'une quinte descendante.

3. Troisième étape: tourner à nouveau le disque d'une autre quinte descendante, ce qui place le $\mathrm{Ma}^{\mathrm{l}} \mathrm{du}$ disque mobile sur le $\mathrm{Ni}^{\prime}$ (Sib) de la partie fixe.

4. Quatrième étape: procéder de même; le $\mathrm{Ma}^{1} \mathrm{du}$ disque mobile est alors sur le $\mathrm{Ga}^{1}$ (Mib) de la partie fixe.

5. Cinquième étape: prendre le mode mineur relatif de Mib, ce qui donne la gamme de Do mineur.

Résumé: On a ainsi modulé de Do majeur à Do mineur par quatre quintes descendantes successives et opéré une transposition modale à la gamme mineure relative. Les positions tonales indiquées sur la partie fixe, en face des repères du disque mobile, indiquent la gamme de Do mineur en partant de Sa (Do) de la partie fixe : il est à noter que les positions enharmoniques de Do $(\mathrm{Sa}), \operatorname{Ré}\left(\operatorname{Re}^{3}\right)$, et Sol $\left(\mathrm{Pa}^{3}\right)$ ont été abaissées d'un comma.

Nous avons étudié expérimentalement l'enchaînement et la modulation de Do majeur à Do mineur:

1. en intonation juste, en abaissant le Do, Ré, et Sol d'un comma ;

2. en intonation juste, sans changement de la tonique de ces deux gammes ;

3. en utilisant le tempérament égal.

34. En fait le système grama-murcchana se sert, pour explorer les échelles musicales, de six échelles fondamentales (grama-s): Sa-grama, Ma-grama, et leurs deux versions altérées - utilisant antara gandhara ou antara gandhara et kakali nishad. Il est hors de notre propos d'exposer en detail la signification acoustique et "grammaticale" de ces grama-s, qui satisfont différemment aux exigences de la musique modale et de la musique harmonique. 


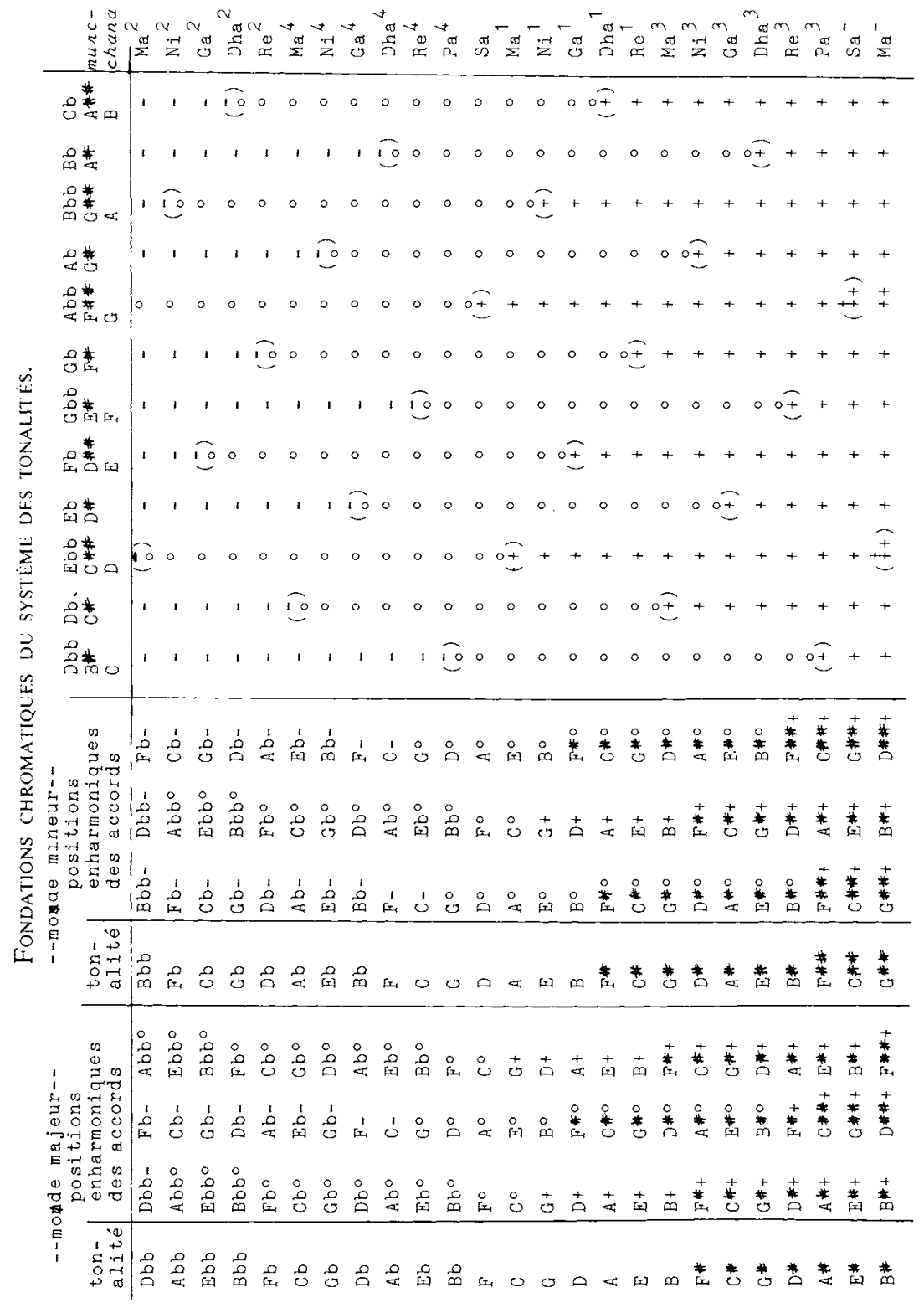


Disque MOBILE DE GRAMA.

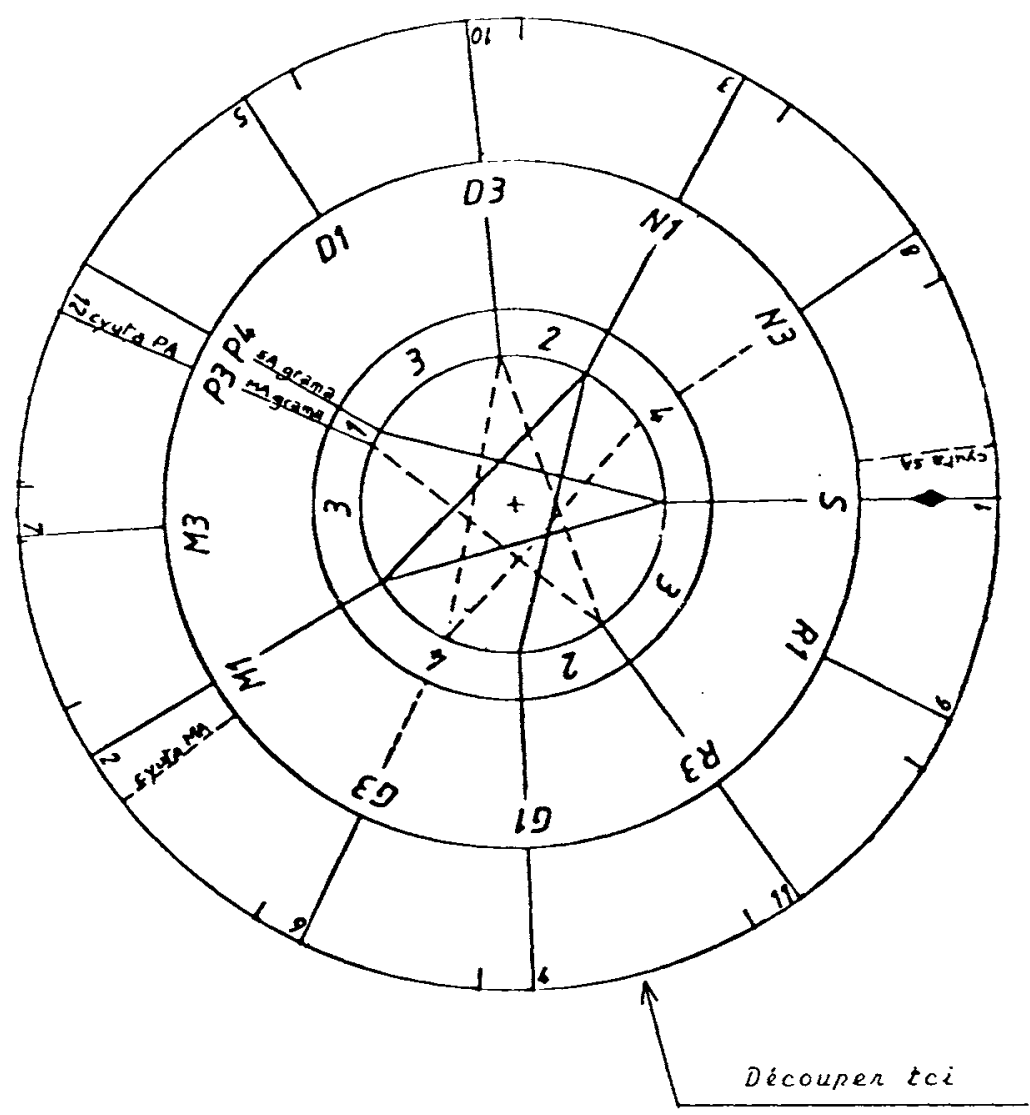



Graphe fixe des Shrutis.

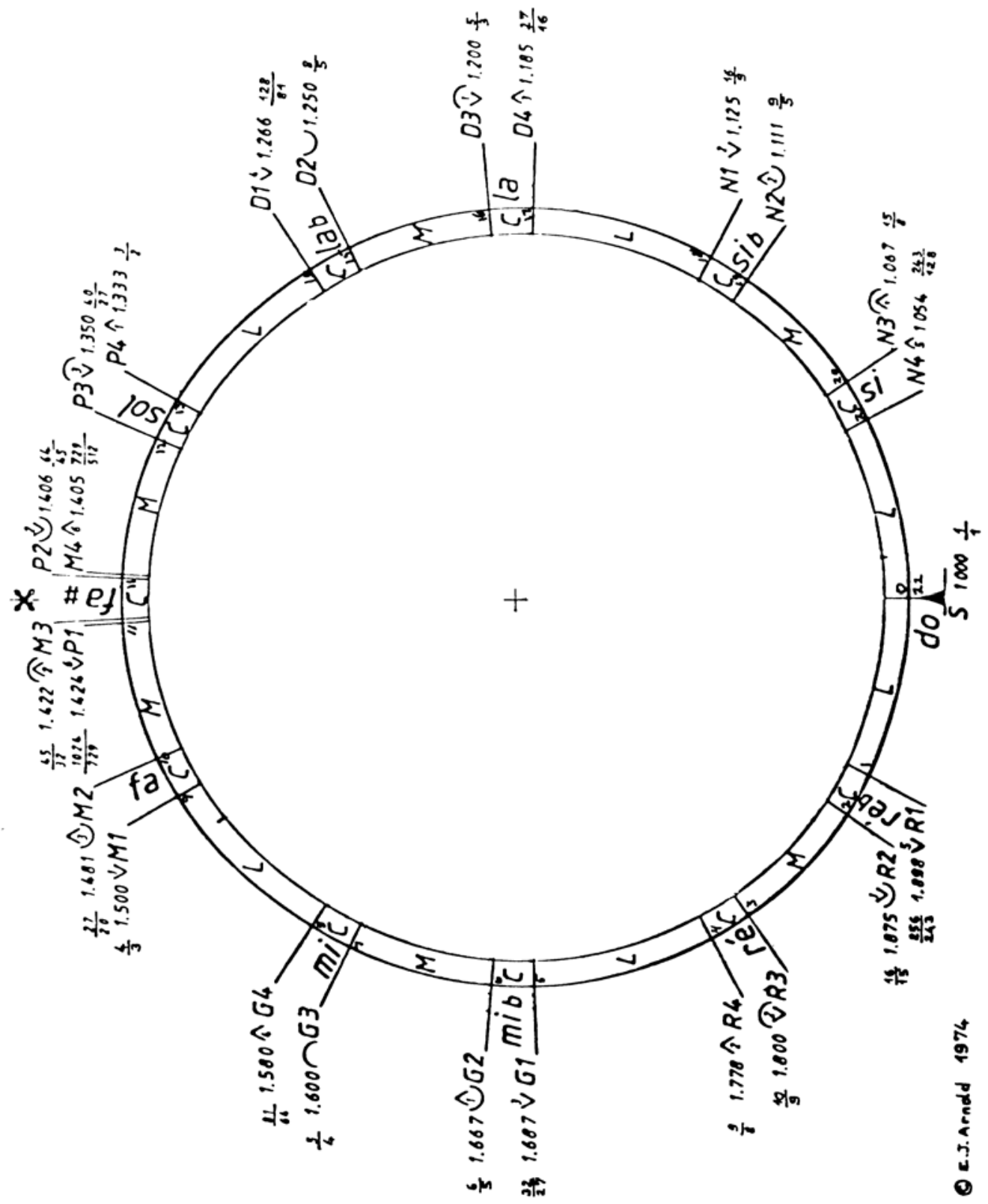


La vérification auditive a montré que l'effet de cet enchaînement d'accords ou modulation des gammes est nettement renforcé dans le premier cas, conformément aux exigences du système gramamurcchana. Cela s'applique aussi aux autres enchaînements ou modulations.

2. Les gammes majeure et mineure en intonation juste, et leurs rapports aux échelles fondamentales Ma-grama et Sa-grama

Placer une fois de plus le $\mathrm{Ma}^{1}$ de la partie mobile du modèle sur le Sa de la partie fixe pour obtenir la gamme majeure de Zarlino. Comme nous l'avons souligné en II.5, la note "critique » de l'échelle fondamentale (grama), le pancam, ou cinquième degré du grama (qui peut être haussé ou baissé d'un comma, déterminant ainsi s'il s'agit de Sa-grama ou Ma-grama) se retrouve à des positions différentes par transposition modale (changement de murcchana), ainsi que par transposition de tonalité (modulation).

Dans le cas de la gamme de Zarlino, en musique harmonique, la note critique devient (Ré) ( $\mathrm{Re}^{3}$ ou $\mathrm{Re}^{4}$ ), et non (La), comme on l'imagine souvent. La note (La), doit jouer le rôle de tierce majeure harmonique (rapport 5/4) dans l'accord de (Fa) majeur, et doit simultanément se trouver à la même position enharmonique pour être à une tierce mineure harmonique $(6 / 5)$ au-dessous de (Do) pour la gamme mineure relative (et son accord de premier degré). Le seul cas où la note (La) devient critique est celui où la relation mélodique (Sol)-(La) doit refléter celle de (Do)-(Ré); c'est-à-dire lorsqu'on a modulé vers (Sol) majeur, ou qu'on est sur le point de le faire. La note (Ré), par contre, doit être haute (9/8) lorsqu'elle appartient à l'accord de (Sol) majeur, et basse (10/9) lorsqu'elle appartient à l'accord de (Ré) mineur. Dans ce dernier cas, elle est en position correcte pour former le quatrième degré de la gamme de (La) mineur.

Ainsi, le (Ré) le plus haut (qui définit l'échelle Sa-grama) est nécessaire pour l'accord de dominante du mode majeur; par contre, le (Ré) le plus bas (qui définit l'échelle Ma-grama) est nécessaire pour l'accord de sous-dominante du mode mineur. Le mode majeur est donc une échelle Sa-gram-ique, tandis que le mode mineur est une échelle Ma-gram-ique.

\section{Positions enharmoniques utilisées lors de modulations}

Si l'on module à partir de la gamme de Zarlino située sur Do (ou sa gamme mineure relative, La) selon douze quintes ascendantes et douze quintes descendantes, on observe qu'il est nécessaire de disposer de douze positions tonales (shruti-s) supplémentaires, dont onze se situent un comma plus bas par rapport aux positions les plus basses du modèle d'origine, et une se situe un comma plus haut que la plus haute.

Le tableau page 32 indique les positions enharmoniques des 
gammes chromatiques associées aux divers accords et tonalités. Les positions tonales médianes, appartenant au cycle des quintes descendantes, sont représentées par le symbole «"». Les positions élevées ou abaissées d'un comma sont représentées, respectivement, par les symboles " $+»$ et $"-»$. Les symboles entre parenthèses correspondent aux notes "critiques" des échelles fondamentales Magrama et Sa-grama (voir II.5).

Dans un groupe de trois lignes horizontales sur le tableau, le nom de la note qui commence la ligne médiane marque la tonalité sur laquelle est située la gamme de Zarlino, les notes de ses accords de $I^{\text {er }}$ et $V^{\text {e }}$ degré, sa gamme mineure relative, et la structure enharmonique de la gamme chromatique qui est "en arrière plan ». Les lignes horizontales immédiatement au-dessus et au-dessous d'une ligne donnée, donnent les positions enharmoniques des autres accords de la gamme sauf celui du VII degré.

Les expériences que nous avons menées avec $M$. Pierre-Yves Asselin, interprétant sur le shruti harmonium des pièces de J.-S. Bach et d'autres compositeurs en intonation pure selon les principes cidessus, laissent penser qu'une recherche pratique dans ce domaine pourrait permettre une audition différente de la musique harmonique. Les compositeurs qui travaillent sur ordinateur auraient tout interêt à utiliser les règles d'analyse du système grama-murcchana pour en mesurer l'effet dans les contextes de l'harmonie classique et contemporaine.

\section{GLOSSAIRE}

antara gandhar : la tierce majeure harmonique de l'échelle fondamentale

(Ma-grama ou Sa-grama).

asavari: mode de La.

bilaval: mode de Do.

bhairao: mode de Do avec seconde et sixte diminuées.

bhairavi: mode de $\mathrm{Mi}$.

cyuta: abaissé d'un comma.

comma : $81 / 80$.

demi-ton chromatique : $25 / 24$.

Dha (dhaivat): sixième degré de la gamme (La).

Ga (gandhar) : troisième degré de la gamme (Mi).

grama: échelle fondamentale définie dans les traités sanscrits anciens. harmonique: ensemble de positions tonales construites à partir de

la tierce majeure harmonique ou de la sixte mineure harmonique au-dessus de la tonique.

kakali nishad: la septième majeure (Si) harmonique de l'échelle fondamentale (Ma-grama ou Sa-grama).

kalyan: mode de Fa.

kaphi: mode de Ré.

khamaj: mode de Sol.

komal: bémol ou "mineur». 
lalita: mode de $\mathrm{Si}$ avec la tierce majeure et la septième majeure. limma : 256/243.

$M a$ (madhyam): quatrième degré de la gamme ( $\mathrm{Fa})$.

marva: mode de Fa avec la seconde mineure.

murcchana: mode ou transposition modale.

$\mathrm{Ni}$ (nishad): septième degré de la gamme (Si).

$\mathrm{Pa}$ (pancham): cinquième degré de la gamme (Sol).

purvi: mode de $\mathrm{Fa}$ avec la seconde et la sixte diminuées.

pythagoricien: ensemble de positions tonales obtenues par cycles de quintes ou quartes.

raga: principe d'organisation musicale, à la base de la musique indienne. Les ragas se différencient par leurs échelles tonales, leurs mouvements mélodiques et les notes "accentuées" ou "étouffées". Re (rishabh): deuxième degré de la gamme (Ré).

sadharana: transposition d'un comma.

$\mathrm{Sa}$ (sadja): tonique ou premier degré de la gamme.

sandhiprakash: le changement de lumière c'est-à-dire l'aube ou le crépuscule.

sangita: la musique.

shastra: tradition écrite.

shruti: 1. Position potentielle d'une note dans l'octave. 2. Intervalle entre deux positions.

shuddha grama : échelle fondamentale d'origine (sans utiliser les notes altérées - vikrit swara-s).

shuddha swara: notes "naturelles". Noter que kaphi est l'ancienne échelle "naturelle» et bilaval la moderne.

sphota: manifestation claire et distincte.

swara: note.

tivra: dièse, ou «majeur ».

todi: mode de Mi mais avec la quarte et la septième augmentées. vadi swara: note de "tension" d'un raga donné.

vikrit swara: note «altérée (terme ancien). Les notes "altérées" sont antara gandhar et kakali nishad.

\section{SUMMARY}

The theory of just intonation based on two fundamental scales (grama-s) described in Bharata's Natya Shastra and other early Sanskrit musicological texts formalizes the relations found in diatonic musical scales with an astounding perspecuity. Only minimal changes to that system are necessary to account for the non-diatonic scales also popular in contemporary Indian music. The paper argues that Bharata's theory provides a better psychoacoustic rational for playing ragas at particular times of the day than the current explanation. The mathematical model of this system of interval relations in diatonic and related scales presented here is a useful tool to study the relations further. Discussion ends on a consideration of implications of the Indian system for harmonic music in just intonation.

Édité et traduit de l'anglais par Bernard Bel Année de rédaction : 1980 